Y. C. Minh and E. F. van Dishoeck, eds.

\title{
Interstellar and Circumstellar PAHs
}

\author{
A. G. G. M. Tielens, C. van Kerckhoven ${ }^{1}$, E. Peeters, and S. Hony ${ }^{2}$ \\ SRON/Kapteyn Astronomical Institute, PO Box 800, 9700 AV \\ Groningen, The Netherlands
}

\begin{abstract}
ISO has shown that the mid-IR spectra of almost all sources are dominated by narrow emission features collectively known as the UIR bands. These bands are carried by a family of polycyclic aromatic hydrocarbon molecules containing about $50 \mathrm{C}$-atoms. Other classes of molecules such as C-chains as well as non-aromatic groups attached to the PAHs are much less abundant. ISO has revealed that the UIR spectrum is incredibly rich with a large number of weaker features, subfeatures, and shoulders; many of which were not known before. Some examples and the new insights they can provide are described. A systematic study of this spectral structure may well allow us to considerably narrow down the composition of the family of PAHs responsible for the observed emission.
\end{abstract}

\section{Introduction}

The IR spectra of bright sources with associated dust and gas are dominated by relatively broad emission features at $3.3,6.2,7.7,8.6$, and $11.3 \mu \mathrm{m}$, which always appear together. Because the carriers of these bands remained unidentified for almost a decade, these bands have become collectively known as the Unidentified InfraRed (UIR) bands. The identification with an aromatic carrier is now universally accepted (cf. §3) and this name is somewhat of a misnomer. Nevertheless, the abbreviation has stuck and is widely used. The ISO mission was ideally suited to study these emission features: spectroscopically using the SWS spectrometer, ISOPHOT-S, and the ISOCAM-CVF and spatially with ISOPHOT and ISOCAM. This paper reviews these observations and some of the main conclusions (see also Tielens et al. 1999).

The IR emission features dominate the mid-IR emission in circumstellar regions, the local diffuse ISM, bright PDRs, nearby normal galaxies, and starburst regions in galactic nuclei. The carriers, PAH molecules, obviously play a major role in the radiative energy budget of the galaxy, including the gas through the photo-electric effect (Verstraete et al. 1990; Bakes \& Tielens 1994). From a curiosity, the UIR bands have now developed into "mainstream" astronomy and, for example, they are now used to derive redshifts and as a diagnostic to separate AGNs from starbursters in the ULIRG population. Likely, these UIR bands are

\footnotetext{
${ }^{1}$ Institute voor Sterrekunde, K.U. Leuven, Celestijnenlaan 200B, 3001 Heverlee, Belgium

${ }^{2}$ Astronomical Institute, University of Amsterdam, Kruislaan 403, NL-1098 SJ Amsterdam, The Netherlands
} 


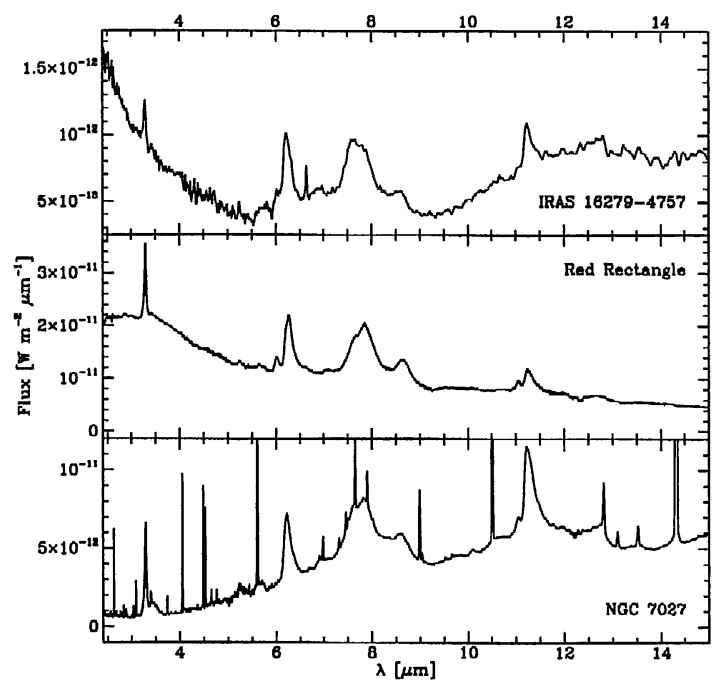

Figure 1. The 3-15 $\mu \mathrm{m}$ emission spectra of the post-AGB objects, IRAS 13428-6232 and the Red Rectangle, and the planetary nebula, NGC 7027 (Hony et al. 2000), illustrating the rich and variable UIR spectrum.

even a characteristic of the ISM of the starbursts in protogalaxies at $z \sim 5$. It is clear that if we want to understand the role of these features in the ecology of galaxies near and far, we have to study the physical and chemical characteristics and the origin and evolution of their carriers in the local universe. Overviewing the field, it seems to us that we have now reached a stage where we can leave the Linnean world of cataloguing the UIR bands and enter the Darwinistic phase of the molecular universe.

\section{The Ubiquitous UIR Bands}

The SWS spectrometer on ISO has for the first time allowed to get complete 2.5$45 \mu \mathrm{m}$ spectra of a great variety of bright ( $>10 \mathrm{Jy}$ ) sources. Except for deeply embedded protostars and AGB objects, all of these spectra show the UIR bands. As an example, Figure 1 shows three spectra of C-rich post-AGB objects and PN (Hony et al. 2000). Representative SWS spectra of HII regions have been published by Roelfsema et al. (1996), Verstraete et al. (1996), and Peeters et al. (1999). Moutou et al. (1999) show a breathtaking SWS spectrum of the reflection nebula, NGC 7023. PAH emission features in the spectra of Herbig AeBe stars are shown in van Kerckhoven et al. (2000). Several of the brighter nuclei of starburst galaxies (e.g. M82, NGC 253, Circinus) have been observed in the mid IR by SWS as well and show bright UIR bands (cf. Rigopoulou et al. 1999).

Much fainter sources ( $>0.1 \mathrm{Jy}$ ) have been observed spectroscopically by ISOPHOT and ISOCAM. Besides bright PDRs such as those associated with HII regions (M17, Henning et al. 1998) and reflection nebulae (NGC 7023, Boulanger 
et al. 1998a), at this level, it becomes possible to measure the mid-IR spectrum of the IR cirrus (Mattila et al. 1996) and surfaces of dark clouds (Boulanger et al. 1998b; Lemke et al. 1998) illuminated by the average interstellar radiation field. In agreement with earlier suggestions (Puget et al. 1985) based upon broad- and narrow-band photometry by IRAS (Low et al. 1984) and the AROME balloon mission (Giard et al. 1994; Ristorcelli et al. 1994), these spectra unambigously demonstrate that the mid-IR spectrum of the ISM is dominated by the UIR bands. This is not only true for the Milky Way but, as demonstrated by the galaxy survey by Helou (1999), is a general characteristic of the ISM in galaxies. A particularly nice example is given by the mapping of the IR spectrum in the edge-on "twin" of the Milky Way, NGC 891 (Mattila et al. 1999). Similar studies of (regions in) nearby bright galaxies have been performed for the LMC (Contursi et al. 1998) and M31 (Cesarsky et al. 1998). It is also possible to spectroscopically survey the nuclei of nearby $(z<0.3)$ starburst galaxies which all show bright UIR bands (Lutz et al. 1998; Bosselli et al. 1997). While the spectra of AGNs do not show the UIR bands - indeed that has been used as one observational diagnostic to separate true AGNs from starbursts (Genzel et al. 1998; Lutz et al. 1998) - that may merely reflect the high optical depth of the circumnuclear torus surrounding the central monster rather than a genuine absence of the carriers of the UIR bands.

\section{The Aromatic Nature of the Emitters}

As first noticed by Duley \& Williams (1981), the observed UIR spectrum is very characteristic for aromatic species and this is not controversial at all anymore (cf. Allamandola et al. 1989 for a discussion of the spectroscopy of aromatic materials). Nevertheless, in the literature one may still sometimes find the bands attributed to such diverse species/materials as Polycyclic Aromatic Hydrocarbons (PAHs), hydrogenated amorphous carbon (HAC), Quenched Carbonaccous Composites (QCC), or Coal. In judging these assignments, it should be understood that any good spectral match is only provided by the aromatic structures within these materials. Except for the PAHs, these materials generally contain a substantial aliphatic component as well and the spectral characteristics of the latter often dominates their IR spectra. However, when these materials have undergone substantial heat treatment to drive off any heteroatoms such as $\mathrm{O}$ or excessive $\mathrm{H}$ and to graphitize the C-skeleton, their IR spectra can provide great matches to the observed UIR spectrum; reemphasizing the aromatic nature of the carrier of the UIR bands. The spectra of heat-treated coals provide a telling example of this (Guillois et al. 1996). We do note, however, that, while the global observed spectra are consistent with PAH molecules as well aromatic carbonaceous solids, the observed high feature-to-continuum ratio $(\simeq 30$ for the $3.3 \mu \mathrm{m}$ in NGC 1333; Joblin et al. 1996) provides direct support for a molecular carrier (Geballe 1997).

The availability of complete $2.5-45 \mu \mathrm{m}$ spectra of the interstellar UIR bands allows a detailed search for the chemical structure of the carriers. In particular, interesting limits can be placed upon the presence of various sidegroups on the emitting PAHs. The abundance of various sidegroups determined from the observed spectra is summarized in Table 1. Structural formulae for these PAHs 
Table 1. The overwhelming aromatic nature of the UIR bands.

\begin{tabular}{lcc}
\hline Functional group & $\begin{array}{c}\lambda \\
(\mu \mathrm{m})\end{array}$ & fraction $^{a}$ \\
\hline aromatic $(\mathrm{H})$ & 3.3 & 1 \\
aliphatic $\left(\mathrm{CH}_{3}\right)$ & 3.40 & 0.02 \\
hydroxyl $(\mathrm{OH})$ & 2.77 & $<0.002$ \\
amine $\left(\mathrm{NH}_{2}\right)$ & $2.88-2.95$ & $<0.01$ \\
aldehydic $(\mathrm{HCO})$ & 5.9 & $0.006(?)$ \\
nitrile $(\mathrm{C} \equiv \mathrm{N})$ & 4.48 & $<0.01$ \\
acetylenic $(\mathrm{C} \equiv \mathrm{CH})$ & 3.03 & $<0.003$ \\
\hline
\end{tabular}

${ }^{a}$ Fraction relative to aromatic hydrogen

with sidegroups can be found in Geballe et al. (1989; their Figure 3). The overwhelming majority of the edge sites of the aromatic carrier of the UIR bands contain aromatic $\mathrm{H}$ with a minute amount of aliphatic $\mathrm{CH}_{3}$ groups and possibly some aldehydic groups (HCO). This strongly reinforces the overwhelming aromatic nature of the UIR bands emphasized above. Clearly, the carrier of the UIR bands does not consist of aromatic units bound by aliphatic chains in a three dimensional, coal-like or HAC-like structure. In principle, the spectroscopic evidence still allows a carrier consisting of PAH units bonded together in a three dimensional network by physical van der Waals forces. However, two $25 \mathrm{C}$-atom PAHs are bonded only by $0.6 \mathrm{eV}$ and FUV photon absorption will rapidly breakdown such a cluster (Léger \& Puget 1984).

\section{PAH Molecules and the UIR Spectrum}

While the vibrational spectrum of any (almost) exclusively aromatic material can provide a good fit to the observed circumstellar and interstellar UIR spectrum, the UIR bands are generally attributed to small PAH molecules containing 50 C-atoms rather than coal, HAC, or QCC dust grains. The main evidence for this is an argument originally presented by Sellgren (1984) in connection to the mid-IR emission observed far from the illuminating star in reflection nebulae and this point has now been reinforced by the ISO observations of strong UIR bands in the diffuse ISM (Mattila et al. 1996; Boulanger et al. 1998b). Specifically, the observed "color" temperature of the UIR bands in the ISM is $\sim 650 \mathrm{~K}$, while the (observed) radiative equilibrium temperature of interstellar dust is $\simeq 15 \mathrm{~K}$ and "classical" grains will not emit much shortwards of $100 \mu \mathrm{m}$ (Draine \& Anderson 1985). The UIR bands are therefore attributed to "cooling" radiation of small species which because of their limited heat capacity attain some $1000 \mathrm{~K}$ upon absorption of a single FUV photon. The observed color temperature corresponds then to species containing about $50 \mathrm{C}$-atoms absorbing a $\sim 10 \mathrm{eV}$ photon. Thus, the emitters are polycyclic aromatic hydrocarbon molecules with about $50 \mathrm{C}-$ atoms such as hexabenzocoronene $\left(\mathrm{C}_{48} \mathrm{H}_{24}\right)$ and circumcoronene $\left(\mathrm{C}_{54} \mathrm{H}_{18}\right)$. 
Table 2. Carriers of IR emission components.

\begin{tabular}{lcccc}
\hline carrier & IR emission component & $N_{c}$ & $\begin{array}{c}a \\
(\AA)\end{array}$ & $\begin{array}{c}f_{C}{ }^{a} \\
(\mathrm{ppm})\end{array}$ \\
\hline PAHs & UIR bands & $20-100$ & $4-10^{b}$ & 14 \\
PAH-clusters & plateaus & $100-1000$ & $10-20$ & 8 \\
very small grains & $25 \mu \mathrm{m}$ cirrus & $10^{3}-10^{4}$ & $20-30$ & 7 \\
small grains & $60 \mu \mathrm{m}$ cirrus & $\sim 10^{5}$ & 50 & 16 \\
classical grains & $\lambda>100 \mu \mathrm{m}$ & & $\gtrsim_{100}$ & $35^{c} ?$ \\
\hline
\end{tabular}

${ }^{a}$ Abundance of $\mathrm{C}$ locked up in these species in parts per million.

${ }^{b}$ Size corresponds to disk rather than sphere.

${ }^{c}$ The abundance of C locked up in dust grains is very uncertain (cf. Snow \& Witt 1996).

\section{ISO Spectroscopy and Interstellar PAHs}

The flood of high quality ISO spectra that is now becoming available will lead to new insights in the composition, origin and evolution of interstellar PAHs. The first results of this are now emerging and have been reviewed by Tielens et al. (1999). Some spectroscopic results are presented here. Table 1 summarizes the observed aromatic nature of the carriers while Table 2 reviews the abundances of various emission components present in the ISM.

\subsection{The incredibly rich spectrum of interstellar PAHs}

The SWS spectra of bright sources obtained by ISO have revealed the incredible richness of the interstellar and circumstellar UIR spectrum. Besides the wellknown UIR bands at $3.3,6.2,7.7,8.6$, and $11.3 \mu \mathrm{m}$, there is a wealth of weaker features: $3.4,5.25,5.65,6.9,10.5,12.7 \mu \mathrm{m}$ to mention some of them (Fig. 1; cf. Beintema et al. 1996). Moreover, many of the prominent bands show satellite bands (e.g. 6.0,11.0 $\mu \mathrm{m}$ ) or break up into subfeatures (e.g. 7.6 and $7.8 \mu \mathrm{m}$ ). A particularly good example of this spectral richness is also provided by the high S/N , 7-9 $\mu \mathrm{m}$ spectrum of NGC 7023 where in this small wavelength range already about 10 subfeatures can be discerned (Moutou et al. 1999). The 11-15 $\mu \mathrm{m}$ region is another illustration of this spectral extravagance (cf. §5.3).

It should be emphasized that not all sources show all of these emission components at the same time. Indeed, spectral variation is one important characteristic of the UIR spectrum. In particular, all sources show the 5 well-known features but their ratios can vary substantially and the 11.3/7.7 ratio in Fig. 1 provides a good example. More dramatic, some HII regions show a $8.6 \mu \mathrm{m}$ feature and some Herbig AeBe stars show a $3.5 \mu \mathrm{m}$ feature which dwarf the commonly much brighter 7.7 and $3.3 \mu \mathrm{m}$ features, respectively (Roelfsema et al. 1996; Verstraete et al. 1996; van Kerckhoven et al. 1999). More subtle variations are provided by the ratio of the 7.6/7.8 components of the $7.7 \mu \mathrm{m}$ feature among HII regions and planetary nebulae (Beintema et al. 1996; Roelfsema et al. 1996; Peeters et al. 1999). Also, often the features vary relative to each other with distance from the illuminating star within a single object (Geballe et al. 1989; 
Joblin et al. 1996, 2000). These spectral variations make each spectrum unique and imply that the carriers are sensitive to the local physical conditions.

\subsection{Interstellar PAH ions}

While the peak positions of the UIR bands are very characteristic for PAHs, available laboratory spectra of neutral PAHs do not produce a good fit to the data in terms of relative strength of the features. In particular, the $\mathrm{CH}$ out-ofplane bending modes in the $11-15 \mu \mathrm{m}$ region are intrinsically much too strong relative to the CC stretching modes in the 5-8 $\mu \mathrm{m}$ region. From the beginning, this difference has been ascribed to the effects of ionization on the intrinsic strength of these modes (Allamandola et al. 1985). The influence of ionization on the spectral characteristics of PAHs has been studied in the laboratory in low temperature matrices (Hudgins \& Allamandola 1995; Szczepanski \& Vala 1993) and by theoretical quantumchemical calculations (Langhoff 1996; deFrees et al. 1993; Pauzat et al. 1997). Using very elegant molecular beam techniques, the visual and IR spectra of PAH neutrals (Schlemmer et al. 1994; Cook et al. 1996) and cations (Piest et al. 1999; Brechignac \& Pino 1999) have now been reported in the gas phase, supporting both the spectroscopy and the emission mechanism in interstellar PAH models. Calculated spectra of (charged) PAH families are now in very good agreement with the observed interstellar UIR spectrum, both in peak position and in relative strength (cf. Allamandola et al. 1999; Bakes et al. 2000).

The PAHs charge depends on the ratio of the ionization rate to the recombination rate; i.e., $G_{o} T^{0.5} / n_{e}$ with $G_{o}$ the intensity of the ambient radiation field, $T$ the gas temperature, and $n_{e}$ the electron density (Bakes \& Tielens 1994). As a result, the emitted spectrum will depend on the local physical conditions and some of the variations observed (cf. §3) may well reflect variations in the physical conditions, notably $G_{o}$. In particular, variations in the relative strength of the 11.3 and $8.6 \mu \mathrm{m}$ bands with distance from the illuminating star in the NGC 1333 reflection nebula have been ascribed to variations in the degree of ionization of the PAHs involved (Joblin et al. 1996). Similarly, the variations in the 11.3 to the $7.7 \mu \mathrm{m}$ bands in a sample of HII regions has also been attributed to variations in the charge distribution (Roelfsema et al. 1996; Peeters et al. 1999). The great strength of the $11.3 \mu \mathrm{m}$ band in the ISM of the galaxy M31 (Cesarsky et al. 1998) may also reflect charge differences with the local solar neighborhood but now on a galaxy-wide scale.

\subsection{The $\mathbf{C}-\mathbf{H}$ out-of-plane deformation modes}

The 11-15 $\mu \mathrm{m}$ region of the spectrum has long been known to be very characteristic for the edge structure of the emitting aromatic species (Duley \& Williams 1981; Cohen et al. 1985; Allamandola et al. 1989). While IRAS-LRS had already shown the presence of other bands than the strong $11.3 \mu \mathrm{m}$ band, ISO-SWS has really unleashed the full power of IR spectroscopy on this problem. As illustrated by Fig. 2, this spectral region is rich in detail and, in the Red Rectangle, contains bands at 11.0,11.3,11.9, 12.8, 13.6, 14.2, and $14.8 \mu \mathrm{m}$.

This spectral region is characteristic for the $\mathrm{C}-\mathrm{H}$ out-of-plane deformation mode. Due to coupling between the vibrating $\mathrm{H}$-atoms, the exact peak position of these modes depends on the number of directly adjacent $\mathrm{H}$-atoms (i.e., bonded 


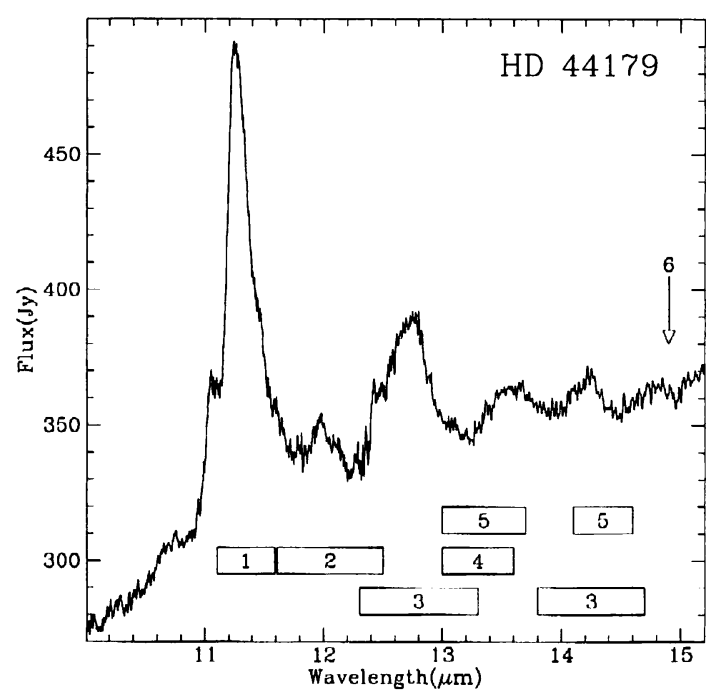

Figure 2. The 10-15 $\mu \mathrm{m}$ spectrum of the Red Rectangle (Hony et al. 2000). Bands in this spectral region are largely due to the $\mathrm{C}-\mathrm{H}$ out-of-plane bending mode whose exact peak position is sensitive to the number of adjacent $\mathrm{H}$-atoms involved. The boxes underneath the spectra give the ranges where neutral PAHs with the indicated number of adjacent $\mathrm{H}$-atoms emit. The arrow labelled 6 indicates the position for Benzene.

to neighboring $\mathrm{C}$ atoms on a ring). The well-known $11.3 \mu \mathrm{m}$ band is attributed to this mode for isolated H's in neutral PAHs. The 11.9 and $12.8 \mu \mathrm{m}$ bands would then be due to 2's and 3's. Now, ionization shifts these ranges for the different number of adjacent H's to shorter wavelengths (Langhoff 1996). The $11.0 \mu \mathrm{m}$ band is thus likely due to the "1" mode in cations and the $11.3 \mu \mathrm{m}$ band might be 2's in PAH ions. However, an origin of the latter in neutral 1's should not yet be disregarded since this mode is so much more intense in neutral PAHs. A systematic study of this wavelength region for $\mathrm{PAH}$ neutrals and cations is presently underway at the Ames laboratory (Hudgins \& Allamandola 2000) and, in combination with the ISO data now becoming available, this will be instrumental in settling these issues (Hony et al. 2000). We also draw attention to the bands at 13.6 and $14.2 \mu \mathrm{m}$. These two weak bands are present in more spectra and signal the presence of 3 and 4 adjacent H's (Hony et al. 2000).

\section{4. $\quad$ Ring bending modes}

Figure 3 shows an emission plateau in the $15-20 \mu \mathrm{m}$ region of two Herbig AeBe stars (van Kerckhoven et al. 2000). The mid-IR spectra of these stars show prominent PAH emission features. The dust continuum emission in the spectra of these stars starts at longer wavelengths than in most other sources and hence these weak features in the $15-20 \mu \mathrm{m}$ region are more readily recognized here. However, this plateau can be recognized in spectra of other sources as well. Some spectra show a distinct feature at $16.4 \mu \mathrm{m}$ superimposed on this plateau (cf. Fig. 3; van Kerckhoven et al. 2000). 


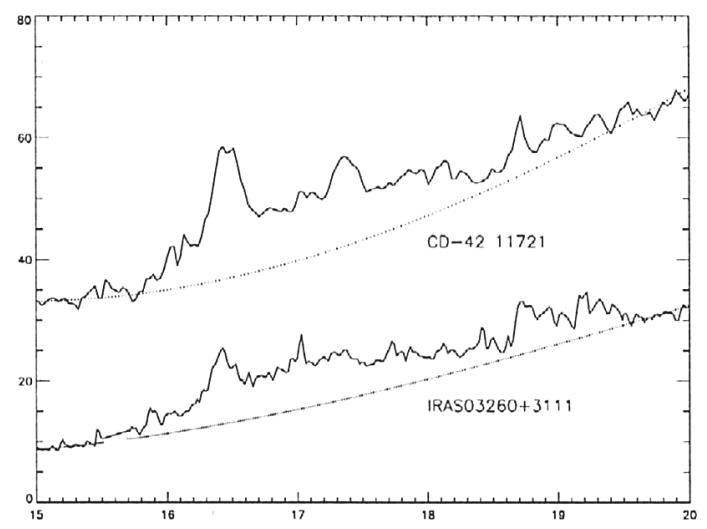

Figure 3. The 15-20 $\mu \mathrm{m}$ spectrum of two Herbig AeBe stars shows excess emission over the continuum. Various new UIR bands are present which can be attributed to ring bending modes (van Kerckhoven et al. 2000).

The far-IR modes of PAHs have been studied in the laboratory by Moutou et al. (1996) and by Hudgins \& Allamandola (2000). In the mid-IR, the vibrational spectrum reflects nearest neighbor vibrations and as a result IR modes occur at very similar wavelengths. In contrast, in the far-IR, emission is due to $\mathrm{C}-\mathrm{C}-\mathrm{C}$ stretching modes which cause in-plane and out-of-plane distortions of the $\mathrm{C}$-skeleton and hence these modes are more molecule specific. In particular, the $16.4 \mu \mathrm{m}$ band may signal the presence of asymmetry in the molecular structure such as associated with the presence of a pentagon (van Kerckhoven et al. 2000). This wavelength region has great potential for the identification of molecular structures which dominate the interstellar PAH family.

\section{Molecular Darwisnism}

As a molecular Darwinist, one may consider the molecular universe from two opposite perspectives: every molecule has a niche and each niche has its molecule. As is abundantly clear from the results of the ISO satellite, the niche for PAHs is the whole universe (cf. §2). The abundance of interstellar PAHs is summarized in Table 2.

The available spectra can also be perused for the presence of other classes of molecules (see Table 3). Large hydrocarbon chains are of course of particular interest in view of the suggestion that $\mathrm{C}_{7}^{-}$is the carrier of some of the Diffuse Interstellar Bands (Tulej et al. 1998; but see Sarre, this volume). Acetylenic C-chains will emit between 4.5 and $5 \mu \mathrm{m}$; i.e., the fundamental frequencies of $\mathrm{C}_{5}$ and $\mathrm{C}_{7}$ are at 4.61 and $4.67 \mu \mathrm{m}$ (Heath et al. 1989). SWS spectra of bright PDRs associated with HII regions or PNe do not show a distinct feature at these wavelengths and neither does the IR spectrum of the diffuse ISM (Boulanger et al. $1998 \mathrm{a}, \mathrm{b})$ to a limit of $1.5 \times 10^{-2}$ compared to the PAH emission. The abundance ratio relative to the PAHs is then,

$$
\frac{f_{\text {chains }}}{f_{\mathrm{PAHs}}}=1.5 \times 10^{-2} \frac{N_{c}(\mathrm{PAHs})}{N_{c} \text { (chains) }},
$$


Table 3. Abundances of large interstellar molecules.

\begin{tabular}{|c|c|c|c|}
\hline Species & Niche & $\begin{array}{l}\text { Abundance } \\
\left(\mathrm{H}^{-1} \text { atom }^{-1}\right)\end{array}$ & $\begin{array}{c}f_{C}^{a} \\
(\mathrm{ppm})^{b}\end{array}$ \\
\hline PAHs & The Universe & $3 \times 10^{-7}$ & 14 \\
\hline C-Chains & Diffuse ISM and PDRs ${ }^{c}$ & $<2 \times 10^{-8}$ & $<2 \times 10^{-1}$ \\
\hline $\mathrm{C}_{7}^{-}$ & 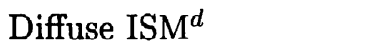 & $8 \times 10^{-10}$ & $6 \times 10^{-3}$ \\
\hline $\mathrm{C}_{60}$ & Diffuse ISM $^{e}$ & $1.5 \times 10^{-8}$ & 1 \\
\hline
\end{tabular}

${ }^{a}$ Abundance of $\mathrm{C}$ locked up in these species.

${ }^{b}$ Parts per million.

${ }^{c}$ For comparison, the abundance of $\mathrm{C}$-chains with more than 3 heavy atoms is $10^{-8}$ in dark clouds (Irvine et al. 1987).

${ }^{d}$ The $\lambda 6270 \AA$ DIB has been attributed to $\mathrm{C}_{7}^{-}$(Tulej et al. 1998). The reported abundance is taken from the DIB study.

${ }^{e}$ Two absorption bands in the far-red have been attributed to $\mathrm{C}_{60}^{+}$requiring an abundance of $10^{-8}$ (cf. Ehrenfreund \& Foing 1997).

with $N_{c}$ the number of C-atoms per carricr. Adopting 10 and 50 for chains and PAHs, respectively, the abundance of chains as a class is small compared to that of the PAHs (0.075; Table 3). The abundance of $\mathrm{C}_{7}^{-}$in the diffuse ISM ${ }^{1}$ (Table 3 ) derived from the $\lambda 6270 \AA$ DIB is $8 \times 10^{-10}$ and this would imply an IR flux in the $4.5-5 \mu \mathrm{m}$ region of $5 \times 10^{-5}$, comfortably less than the observed upper limit of $3 \times 10^{-3}$ in the diffuse ISM. Thus, $\mathrm{C}_{7}^{-}$cannot be excluded as the carrier of the $\lambda 6270 \AA$ DIB from the IR spectra. Clearly, electronic spectroscopy is a much more sensitive tool for the study of molecular species in the ISM than the IR emission.

Now, the $6270 \AA$ band attributed to $\mathrm{C}_{7}^{-}$is a weak DIB and more stringent limits are obtained when considering all the DIBs. Summing up the absorption due to all DIBs ( $W$ (DIBs) $/ E_{B-V} \simeq 17 \AA / \mathrm{mag}$ ), the strength of the IR emission of the DIB carriers relative to the IR emission of the PAHs is,

$$
\frac{F_{I R}(\mathrm{DIB})}{F_{I R}(\mathrm{PAHs})}=7 \times 10^{-3}\left(\frac{0.1}{f_{o s c}}\right) N_{c},
$$

where we adopted an oscillator strength, $f_{\text {osc }}$, of 0.1 . From this, we can draw a few conclusions. If we ascribe all of the DIB absorption to carbon chains with typically $15 \mathrm{C}$-atoms and $f_{o s c}=0.05$, the total IR emission will be $20 \%$ of that of the PAHs; considerably more than the upper limit in the $4.5-5 \mu \mathrm{m}$ region. There are weak UIR bands at 5.2 and $5.6 \mu \mathrm{m}$ at $\simeq 4 \%$ of the total PAH emission, which within the PAH model are attributed to weak combination bands and overtones of the CH out-of-plane, in-plane and aromatic C-C bands (Allamandola et al. 1989). Even attributing these bands to acetylenic chains instead would not suffice to explain all of the DIB absorption. To phrase the DIB-UIR problem in yet a slightly different way, attributing the DIB absorption to a subset of PAHs

\footnotetext{
${ }^{1}$ Note that Tulej et al. (1998) quote a too large required abundance for the carrier of this band
} 
would imply that about $35 \%$ of the PAHs are involved (i.e. in the form of ions or radicals; cf. Salama et al. 1996).

It is not too surprising that $\mathrm{C}$-chains are not very abundant in the diffuse ISM since such relatively small species are photochemically speaking rather fragile in the ISM. In that respect, larger C-rings, possibly bicyclic or tricyclic, might be better candidates for the carriers of the DIBs. But the same abundance limits would apply as derived above for the chains. From a darwinistic point of view, in terms of the IR emission, it may be best to search for small chains in environments where they are rapidly formed such as C-rich stellar outflows rather than in the diffuse ISM.

$\mathrm{C}_{60}^{+}$has tentatively been identified in the diffuse ISM through two far-red DIBs (Ehrenfreund \& Foing 1997). Moutou and Sellgren and their collaborators have taken the Darwinistic approach and looked specifically for the IR signature of $\mathrm{C}_{60}$ and its cation in their spectra (Moutou et al. 1999). They arrive at upper limits of $0.3 \%$ of the elemental carbon. While still in agreement with the DIB assignment, this is much less that the PAH abundance (5\%; cf. Table 3).

\section{PAHs and Chemistry}

\subsection{Formation of interstellar PAHs}

Chemically speaking, PAHs are a general characteristic of high temperature pyrolysis and combustion chemistry. This has been recognized and a number of studies on the origin and evolution of interstellar PAHs start PAH formation in the high temperature, high density ejecta of asymptotic giant branch stars (Frenklach \& Feigelson 1989; Tielens 1990; Cherchneff et al. 1992). The conditions in these outflows resemble those in terrestrial sooting environments except that the $\mathrm{H}$ abundance is higher while all the oxygen is locked up in CO. In these environments, PAHs are the molecular intermediaries in the soot formation process. Detailed models have been constructed and meet with some success (cf. Cherchneff \& Cau 1999). On a galactic scale, AGB stars may be the dominant producers of interstellar PAHs (Latter 1991). That PAHs are indeed formed in such environments has been very elegantly demonstrated by Messenger et al. (1998) who crushed a graphitic stardust grain isolated from a meteorite and discovered PAH-stowaways with a carbon isotope ratio similar to that of the stardust-carrier and distinctly different from the terrestrial value. As a variant on this theme, some models form interstellar PAHs by shattering interstellar carbonaceous grains in grain-grain collisions in shocks. This is a very efficient process and detailed models can explain the observed PAH abundance (Jones et al. 1996). Finally, models for the formation of large molecules in situ through gas phase ion-molecule or neutral-neutral reactions have also been constructed (Bettens \& Herbst 1996). These require very favorable assumptions in terms of the (photo)stability of the small molecular intermediaries $(<20 \mathrm{C}$-atoms) and therefore have not been widely accepted.

\subsection{Chemistry of interstellar PAHs}

A number of studies have appeared concerning the chemistry of interstellar PAHs. Many of these concentrate on the stability of PAHs in the intense ra- 
diation field of PhotoDissociation Regions (PDRs). Peripheral H-loss has been considered by Tielens et al. (1987), Geballe et al. (1989) and Allain et al. (1989) and, while the details differ somewhat, typically small PAHs $(\simeq 25 \mathrm{C}$-atoms) rapidly lose their H's. These models have been extended to consider the loss of aliphatic (e.g. $\mathrm{CH}_{3}$ ) and other sidegroups (Joblin et al. 1996). For typical PDR conditions, species smaller than some $85 \mathrm{C}$-atoms will lose any such sidegroup within $10^{5} \mathrm{yr}$. These models have been successfully applied to explain observed variations in the 3.4-to-3.3 $\mu \mathrm{m}$ ratio in individual PDRs. More recent studies have attributed the $3.4 \mu \mathrm{m}$ band to SPAHs (i.e. Superhydrogenated PAHs with an additional $\mathrm{H}$ ) and the observed variations to (photo)chemistry involving this extra H (Schutte et al. 1993; Sloan et al. 1997; Snow et al. 1998; Bauschlicher 1999). However such an additional $\mathrm{H}$ is bound by only $1.5 \mathrm{eV}$ and is immediately lost upon photon absorption by any PAH smaller than some $100 \mathrm{C}$-atoms. The calculated SPAH abundance at the surface of a PDR is then only $5 \times 10^{-3}$. Further theoretical and experimental studies are required to settle these issues. Finally, interstellar PAHs can also influence the chemistry of other species in the gas phase. In particular, small PAHs can be important in the charge balance of diffuse clouds, dark clouds, and PDRs (Lepp \& Dalgarno 1988; Lepp et al. 1988; Bakes \& Tielens 1998).

\section{Summary}

ISO observations have shown that the UIR bands dominate the mid-IR spectra of almost all objects. The spectra show a rich variety of features; besides the well known $3.3,6.2,7.7,8.6$, and $11.3 \mu \mathrm{m}$ bands there are plethora of weaker features, shoulders, and subfeatures. The detailed spectra of UIR bands vary from one source to another and often also within one nebula. Generally, these variations are small although in a few cases the spectra are radically different.

The UIR bands are very characteristic for aromatic hydrocarbon species. The ISO spectra allow us to place very stringent constraints on the presence of various side groups and the carrier is overwhelmingly aromatic. The strong mid-IR emission observed in the diffuse ISM and in nebulae far from illuminating stars implies that the carrier is small ( $\simeq 50 \mathrm{C}$-atoms), transiently heated through the absorption of a single UV photon. Thus, a PAH molecule. While the abundance of $\mathrm{C}$ in the form of PAH molecules is about $14 \mathrm{ppm}$, about $45 \mathrm{ppm}$ is in species which fluctuate (to some extent) in temperature (i.e. all grain sizes $<50 \AA$ ). The ISO spectra also allow us to search for other interesting molecular species such as $\mathrm{C}$-chains and $\mathrm{C}_{60}$ and interesting limits have been derived. By far, PAHs dominate the molecular universe in the diffuse ISM!

ISO has allowed us to study the UIR spectrum in great detail and a number of new features have been observed, including for the first time, bands in the 15$20 \mu \mathrm{m}$ region due to $\mathrm{C}-\mathrm{C}-\mathrm{C}$ in- and out-of-plane ring bending modes. While no identifications with specific molecules can be made at this point, this wavelength region holds great promise for the future. It is clear that detailed analysis of the spectra obtained by ISO will enable us to constrain the emitting PAH family greatly.

Acknowledgments. We gratefully acknowledge Lou Allamandola, Francois Boulanger, Diego Cesarsky, Pierre Cox, George Helou, Doug Hudgins, Chris- 
tine Joblin, Kimmo Lehtinen, Dieter Lutz, Kalevi Mattila, Claire Moutou, Pjotr Roelfsema, Kris Sellgren, Henrik Spoon, Christoffel Waelkens, and Rens Waters who shared their insight, data and preprints with us. Special thanks go to the ISO-SWS instrument teams at Groningen, Leuven, and Vilspa for help in the data reduction.

\section{References}

Allain, T., Leach, S., \& Sedlmayr, E. 1996, A\&A, 305, 616

Allamandola, L.J., Hudgins, D.M., \& Sandford, S.A. 1999, ApJ, 511, L115

Allamandola, L.J., Tielens, A.G.G.M., \& Barker, J.R. 1985, ApJ, 290, L25 1989, ApJS, 71, 733

Bakes, E.L.O. \& Tielens, A.G.G.M. 1994, ApJ, 427, 822 1998, ApJ, 499, 258

Bakes, E.L.O., et al. 2000, in prep

Bauschlicher, C.W. 1999, ApJ, 509, L125

Beintema, D.A., et al. 1996, A\&A, 315, L369

Bettens, R.P.A. \& Herbst, E. 1996, ApJ, 468, 686

Bosselli, A. et al. 1997, A\&A, 324, L13

Boulanger, F., Boissé, P., Cesarsky D., \& Ryter, C. 1998a, A\&A, 339, 194

Boulanger, F., et al. 1998b, in Star Formation with ISO, eds. J.L. Yun \& R. Liseau (ASP: San Francisco), 132, 15

Brechignac, P. \& Pino, T. 1999, A\&A, 343, L49

Cesarsky, D., Lequeux, J., Pagani, L., Ryter, C., Loinard, L., \& Sauvage, M. 1998, A\&A, 337, 35

Cherchneff, I., Barker, J.R., \& Tielens, A.G.G.M. 1992, ApJ, 401, 269

Cherchneff, I. \& Cau, P. 1999, in AGB stars, eds. T. Le Bertre et al. (ASP: San Francisco), 251

Cohen, M., Tielens, A.G.G.M., \& Allamandola, L.J. 1985, ApJ, 299, L93

Contursi, et al. 1998, A\&A, 336, 662, 15

Cook, D.J., et al. 1996, Nature, 380, 227

deFrees, D.J., Miller, M.D., Talbi, D., Pauzat, F., \& Ellinger, Y. 1993, ApJ, 408, 530

Draine, B.T. \& Anderson, N. 1985, ApJ, 292, 494

Duley, W.W. \& Williams, D.A. 1981, MNRAS, 196, 269

Ehrenfreund, P. \& Foing, B. 1997, Adv. Space Res., 19, 1033

Frenklach, M. \& Feigelson, E.D. 1989, ApJ, 341, 372

Geballe, T.R. 1997, in From Stardust to Planetesimals, eds. Y. Pendleton \& A.G.G.M. Tielens (ASP: San Francisco), 119

Geballe, T.R., Tielens, A.G.G.M., Allamandola, L.J., Moorhouse, A., \& Brand, P.W.J.L. 1989, ApJ, 341, 278

Genzel R., et al. 1998, ApJ, 498, 579

Giard, M., Lamarre, J.M., Pajot, F., \& Serra, G. 1994, A\&A, 286, 203

Guillois, O., Nenner, I., Papoular, R., \& Reynaud, C. 1996, ApJ, 464, 810

Heath, J.R., Cooksy, A.L., Gruebele, M.H.W., Schmuttenmaer, C.A., \& Saykally, R.J. 1989, Science, 244, 564

Helou, G. 1999, in The Universe as seen by ISO, eds. P. Cox, M. Kessler (ESA: Noordwijk), ESA SP 427, 797

Henning, Th., Klein, R., Launhardt, R., Lemke, D., \& Pfau, W. 1998, A\&A, 332,1035 
Hony, S., et al. 2000, in prep

Hudgins, D. \& Allamandola, L.J. 2000, in prep

1995, J. Phys. Chem., 99, 3033

Irvine, W.M., Goldsmith, P.F., \& Hjalmarson, $\AA .1987$, in Interstellar Processes, eds. D.J. Hollenbach \& H.A. Thronson, jr. (Reidel: Dordrecht), 561

Joblin, C., Tielens, A.G.G.M., Allamandola, L.J., \& Geballe, T.R. 1996, ApJ, 458,610

Joblin, C., Bregman J., \& Tielens, A.G.G.M. 2000, in prep

Jones, A.P., Tielens, A.G.G.M., \& Hollenbach, D.J. 1996, ApJ, 469, 740

Langhoff, S.R. 1996, J. Phys. Chem., 100, 2819

Latter, W.B. 1991, ApJ, 377, 187

Léger, A. \& Puget, J.L. 1984, A\&A, 137, L5

Lemke, D., et al. 1998, A\&A, 331, 742

Lepp, S. \& Dalgarno, A. 1988, ApJ, 324, 553

Lepp, S., et al. 1988, ApJ, 329, 418

Low, F., et al. 1984, ApJ, 278, L19

Lutz, D., Spoon, H.W.W., Rigopoulou, D., Moorwood, A.F.M., \& Genzel, R. 1998, ApJ, 505, L103

Mattila, K., et al. 1996, A\&A, 315, L353

Mattila, K., Lehtinen, K., \& Lemke, D. 1999, A\&A, 342, 643

Messenger, S. 1998, ApJ, 502, 284

Moutou, C., Léger, A., \& d'Hendecourt, L. 1996, A\&A, 310, 297

Moutou, C., Sellgren K., Verstraete, L., \& Léger, A. 1999, A\&A, 347, 949

Pauzat, F., Talbi, D., \& Ellinger, Y. 1997, A\&A, 319, 318

Peeters, E., et al. 1999, in The universe as seen by ISO, eds. P. Cox \& M. Kessler (ESA: Noordwijk), ESA SP-427, 739

Piest, H., von Helden, G., \& Meijer, G. 1999, ApJ, 520, L75

Puget, J.L., Léger, A., \& Boulanger, F. 1985, A\&A, 142, L19

Rigopoulou, D., et al. 1999, in The universe as seen by ISO, eds. P. Cox \& M. Kessler (ESA: Noordwijk), ESA SP-427, 833

Ristorcelli, I., et al. 1994, A\&A, 286, L23

Roelfsema, P.R., et al. 1996, A\&A, 315, L289

Salama, F., Bakes, E.L.O., Allamandola, L.J., \& Tielens, A.G.G.M. 1996, ApJ, 458,621

Schlemmer, S., et al. 1994, Science, 265, 1686

Schutte, W.A., Tielens, A.G.G.M., \& Allamandola, L.J. 1993, ApJ, 415, 397

Sellgren, K. 1984, ApJ, 277, 623

Sloan, G.C., et al. 1997, ApJ, 474, 735

Snow, T.P. \& Witt, A. 1996, ApJ, 468, L65

Snow, T.P., Le Page, V., Keheyan, Y., \& Bierbaum, V.M. 1998, Nature, 391, 259

Szczepanski, J. \& Vala, M. 1993, ApJ, 414, 179

Tielens, A.G.G.M. 1990, in Carbon in the Galaxy, ed. J. Tarter, NASA CP-3061, 59

Tielens, A.G.G.M., et al. 1987, in PAHs and Astrophysics, eds. A. Léger et al. (Reidel: Dordrecht), 273

Tielens, A.G.G.M. et al. 1999, in The Universe as seen by ISO, eds. P. Cox \& M. Kessler (ESA: Noordwijk), ESA SP-427, 579

Tulej, M., Kirkwood, D.A., Pachkov, \& Maier, J.P. 1998, ApJ, 506, L69

van Kerckhoven, C. et al. 1999, in The Universe as seen by ISO, eds. P. Cox \&

M. Kessler (ESA: Noordwijk), ESA SP-427, 421

van Kerckhoven, C., et al. 2000, A\&A, submitted 
Verstraete, L., et al. 1990, A\&A, 237, 436

Verstraete, L., et al. 1996, A\&A, 315, L337

\section{Discussion}

J.M. Greenberg: I have been impressed with your careful treatment of grain destruction processes in the ISM. My question is: have you an estimation of the mean lifetime of a PAH?

A. G. G. M. Tielens: PAHs (as are small dust grains) are not really destroyed by shocks up to $200 \mathrm{~km} \mathrm{~s}^{-1}$ because they couple very well dynamically to the gas and the gas cools down rapidly (i.e. sputtering is unimportant). PAHs can be destroyed in the hot gas of a very young supernova remnant (i.e. $\geq 200 \mathrm{~km}$ $\mathrm{s}^{-1}$ shocks). However, these are much less frequent than the low velocity shocks which may rapidly produce PAHs through shattering of carbonaceous dust grains (Jones et al. 1996).

M. C. McCarthy: To improve the specificity of your observations it would obviously be desirable to unequivocally identify some of these molecules in the microwave region by radio astronomy. The rotational spectra of a number of polar polycyclic aromatic hydrocarbons (pyrole, toran, etc.) are known. What are the prospects for future work in this area?

A.G.G.M. Tielens: Observing the pure rotational spectrum of interstellar PAHs could provide specific molecular identification. However, this is very difficult because of the large partition function for $50 \mathrm{C}$-atom, non-linear molecules. It may be possible to observe the ro-vibrational signature in the low frequency (1-3 THz range) bending modes in bright PDRs (Joblin et al., in prep.) where the emission is much enhanced. HIFI, the heterodyne instrument for FIRST, will spectrally survey this important frequency range in a large variety of objects.

$U$. G. Jørgensen: Comment: The references you gave in your talk for the production of PAHs in carbon stars, are calculations for combinations of gas pressure and temperature which are not in agreement with model atmospheres. No selfconsistent model atmospheres for carbon stars predicts formation of PAHs. In hydrostatic models (i.e. the photophere) the temperature is never low enough for PAH formation, and in hydrodynamic models (i.e. the wind) the relevant timescales are too short for PAH formation.

A. G. G. M. Tielens: The exploratory models by Frenklach \& Feigelson (1989) and by Cherchneff et al. (1992) use a simplified description of the gas properties in the extended atmosphere of AGB stars, concentrating on the chemistry. No $\mathrm{PAH}$ or dust formation is expected in the photosphere itself. These models resulted in very low PAH yields $\left(\leq 10^{-4}\right.$ of the $\left.\mathrm{C}\right)$. It is now thought that the shocks in this layer play an essential role in PAH formation (and dust nucleation) by providing a warm and dense environment for periods of years at relatively high heights above the photosphere. It should of course be realized that the models referenced to in this question and answer are only an approximation. Reality is that dust formation does occur in these outflows and that has to go through a nucleation phase. 